\title{
Pay now or pay (more) later: Tracking the costs of hepatitis $\mathrm{C}$ infection
}

\author{
Robert P Myers MD MSc, Samuel S Lee MD
}

$\mathrm{C}$ hronic infection with the hepatitis $\mathrm{C}$ virus (HCV) affects 180 million individuals globally (1), and nearly $1 \%$ of Canadians (2). In Canada, its prevalence is increasing due to new infections and immigration from endemic countries, which outpace deaths and cases of spontaneous and treatment-induced viral clearance. Many reports based on mathematical models (2-4) and, more recently, population-based studies (5), suggest that we are in the midst of an epidemic of HCV-related complications that is projected to continue for at least the next decade. Chronic HCV infection has now emerged as the major indication for liver transplantation in most centres, with a growing number of cases complicated by hepatocellular carcinoma.

The report by Krajden et al (6) in the present issue of The Canadian Journal of Gastroenterology (pages 717-726) is an important addition to the literature illustrating the burden of HCV infection in our country. By linking multiple administrative databases to capture the use of inpatient and outpatient heath services, HCV laboratory testing and prescription pharmaceuticals, the authors provided a detailed snapshot of recent (between 1997 and 2004) spending on direct HCV-related care from the perspective of a provincial payer. The authors should be commended for this major undertaking, which provides valuable data for health resource planning and future costeffectiveness analyses of novel antiviral therapies. Major strengths of this study include the following:

1. Its population-based nature, which facilitated the identification of the majority of $\mathrm{HCV}$-infected patients in British Columbia (BC);

2. The calculation of costs in discrete disease stages (ie, the initial phase, the late phase associated with liver-related complications and the 12 months preceding death from any cause);

3. The inclusion of an HCV-negative control group permitting calculation of 'net' costs directly attributable to HCV; and

4. The authors' rigorous attempts to match HCV antibodypositive patients with controls for various demographic factors (eg, socioeconomic status) and comorbidities (eg, HIV, mental illness and substance abuse), which are more prevalent and have a major impact on costs in this at-risk population.

Ironically, the study's major limitations are related to the same features. Specifically, the data did not include several populations with a high prevalence of HCV infection (eg, Aboriginals and prison inmates), and approximately 15\% of eligible patients were excluded due to missing personal health identifiers (eg, the homeless). Second, the anti-HCV-negative control group was restricted to individuals tested for HCV; these patients undoubtedly differ from the general population due to potential risk factors for HCV acquisition and/or the presence of non-HCV-related liver disease that may have prompted testing. Third, a significant proportion of cases (from approximately $5 \%$ to $20 \%$ depending on disease phase) could not be matched with controls due to their profound vulnerabilities (eg, HIV, substance abuse, mental illness and other comorbidities). Mismatching of these characteristics may explain the significant incremental costs of hospital admission among HCV-positive patients (approximately \$775/year) during the initial phase of infection when HCV-related hospitalization is unexpected. Finally, the cost estimates in this study were clearly underestimates due to incomplete capture of HCV-positive cases (see above), the costs of virological testing, medications for the treatment of HIV, cancer and transplantation, and the indirect costs of chronic HCV infection (eg, reduced productivity and premature mortality).

In spite of these limitations, the study by Krajden et al has several important findings that deserve mention. Notably, the direct costs attributable to $\mathrm{HCV}$ are not inconsequential - the BC government spent an estimated $\$ 1,850 /$ person/year and $\$ 6,000 /$ person/year on direct HCV-related care during the initial and late phases, respectively (net costs during the pre-death phase were similar among HCV-positive cases and controls). Nevertheless, these per patient expenditures pale in comparison with those directed toward HIV care (7). Despite a four- to fivefold greater prevalence of HCV than HIV, the authors estimated that BC spends approximately $\$ 140$ million/year on both conditions (6). Although the reasons behind this discrepancy are multifactorial, it likely relates, in part, to differential access to antiviral therapy. Whereas approximately two-thirds of HIV costs are related to antiretroviral drugs (7), anti-HCV treatments represented only $0.7 \%$ of the case costs in this report. A major reason for this tiny percentage is the strikingly low proportion (less than 1\%) of HCV-positive individuals treated with antiviral therapy during the study period. Given that curative antiviral treatment was licensed and reimbursed by private third-party payers and BC Pharmacare during almost the entire study period, this represents a surprising and disappointing finding. It may be due to many factors including lack of access (eg, to disease diagnosis, referral to a treating physician and drug coverage), eligibility (eg, contraindications) and patient acceptance. Moreover, among provincial formularies, BC Pharmacare has the most restrictive limitations on antiHCV therapies - including the requirement for serum alanine aminotransferase levels to be greater than 1.5 times the upper

Liver Unit, Division of Gastroenterology, Department of Medicine, University of Calgary, Calgary, Alberta

Correspondence: Dr Robert P Myers, Liver Unit, University of Calgary, 6D22, Teaching, Research and Wellness Building, 3280 Hospital Drive

Northwest, Calgary, Alberta T2N 4N1. Telephone 403-592-5049, fax 403-592-5090, e-mail rpmyers@ucalgary.ca

Received and accepted for publication Ncvember 16, 2010 
limit of normal. Considering the large proportion of patients with comorbidities, we believe that this is a major factor in the low rates of treatment uptake. Although information regarding modes of $\mathrm{HCV}$ infection were unavailable in this database study, previous Canadian studies $(8,9)$ have demonstrated that a history of injection drug use is, by far, the dominant mode of HCV infection. Active drug users are well known to have significant mental health issues and psychosocial barriers that, in many cases, preclude or make anti-HCV therapy extremely complicated. Regardless of the reasons, this very low rate of treatment uptake is clearly suboptimal if we hope to reduce future morbidity and mortality attributable to this disease. Because HCV is generally a slowly progressive disease that requires decades to progress to cirrhosis - supported by the fact that approximately $95 \%$ of patients were in the initial phase of disease, and that antiviral therapy is most effective in patients with mild liver fibrosis - we should strive to maximize the dissemination of antiviral therapies earlier during the course of disease to capitalize on this opportunity. Finally, the study by Krajden et al highlights the important influence of HIV, mental illness and addictions on HCV-related costs. To reduce these costs and improve overall outcomes among HCV-infected

\section{REFERENCES}

1. Lavanchy D. The global burden of hepatitis C. Liver Int 2009;29(Suppl 1):74-81.

2. Remis RS. Modelling the incidence and prevalence of hepatitis C infection and its sequelae in Canada, 2007: Final Report. Ottawa: Public Health Agency of Canada, 2009.

3. Zou S, Tepper M, El Saadany S. Prediction of hepatitis C burden in Canada. Can J Gastroenterol 2000;14:575-80.

4. Davis GL, Alter MJ, El-Serag H, Poynard T, Jennings LW. Aging of hepatitis $\mathrm{C}$ virus (HCV)-infected persons in the United States: A multiple cohort model of $\mathrm{HCV}$ prevalence and disease progression. Gastroenterology 138:513-21,e1-6.

5. Myers RP, Liu M, Shaheen AA. The burden of hepatitis $C$ virus infection is growing: A Canadian population-based study of patients, we must also target these conditions with preventive and treatment strategies.

We are in the midst of an epidemic of HCV-related complications that has been likened to "waking a sleeping giant" due to aging and disease progression of the affected population (5). The study by Krajden et al highlighted the substantial costs of this infection, which will undoubtedly increase in coming years. To 'slay the giant', we must continue to focus our attention on preventive strategies, diagnosis of infected cases and, perhaps most importantly, maximizing the use of therapies directed against HCV and frequently associated conditions.

ACKNOWLEDGEMENTS: Dr Myers is supported by a Clinical Investigator Award from the Alberta Heritage Foundation for Medical Research and a New Investigator Award from the Canadian Institutes for Health Research.

DISCLOSURE: Drs Myers and Lee have received research support and lecture and consultancy fees from ScheringPlough (now Merck \& Co), and Roche, manufacturers of antiviral treatment for hepatitis C.

hospitalizations from 1994 to 2004. Can J Gastroenterol 2008;22:381-7.

6. Krajden M, Kuo M, Zagorski B, Alvarez M, Yu A, Krahn M. Health care costs associated with hepatitis C: A longitudinal cohort study. Can J Gastroenterol 2010;24:717-26.

7. Krentz HB, Auld MC, Gill MJ. The changing direct costs of medical care for patients with HIV/AIDS, 1995-2001. CMAJ 2003;169:106-10.

8. Myers RP, Hilsden RJ, Lee SS. Historical features are poor predictors of liver fibrosis in Canadian patients with chronic hepatitis C. J Viral Hepat 2001;8:249-55.

9. Doucette KE, Robson V, Shafran S, Kunimoto D. Improving access to care by allowing self-referral to a hepatitis $\mathrm{C}$ clinic. Can J Gastroenterol 2009;23:421-4. 


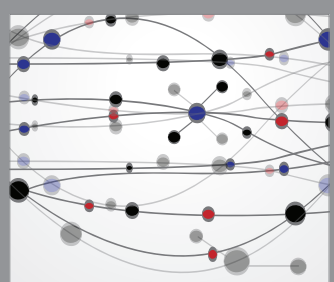

The Scientific World Journal
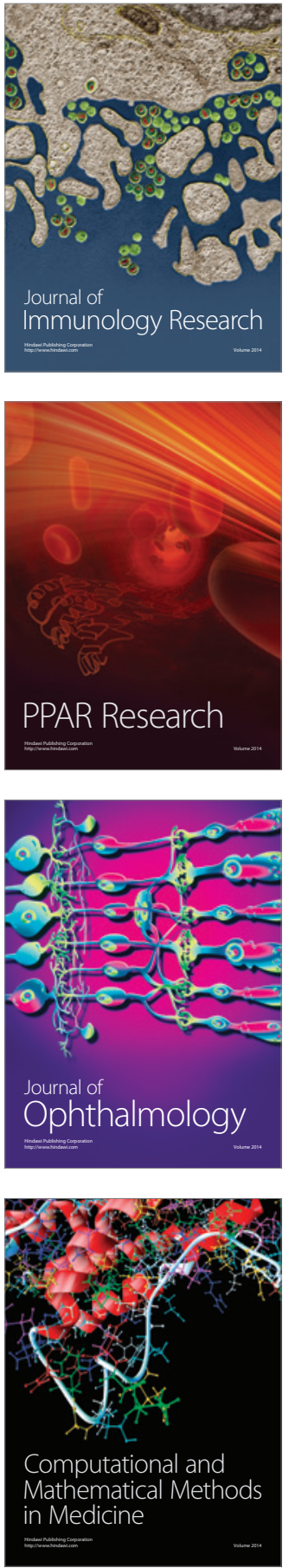

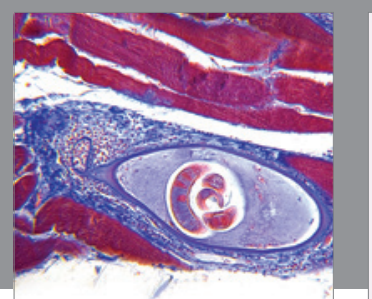

Gastroenterology Research and Practice

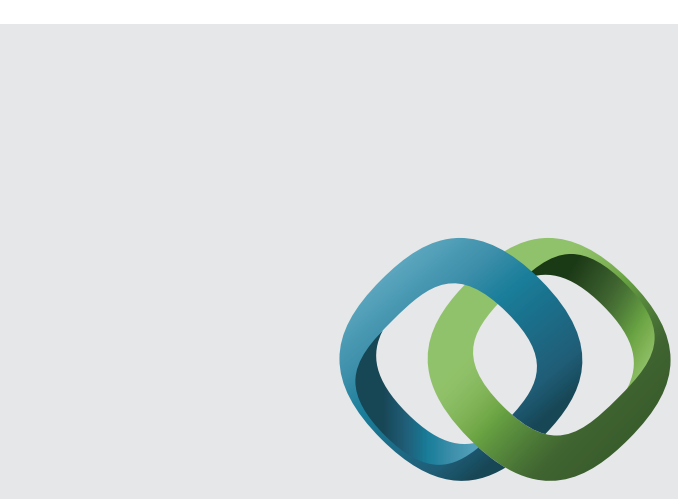

\section{Hindawi}

Submit your manuscripts at

http://www.hindawi.com
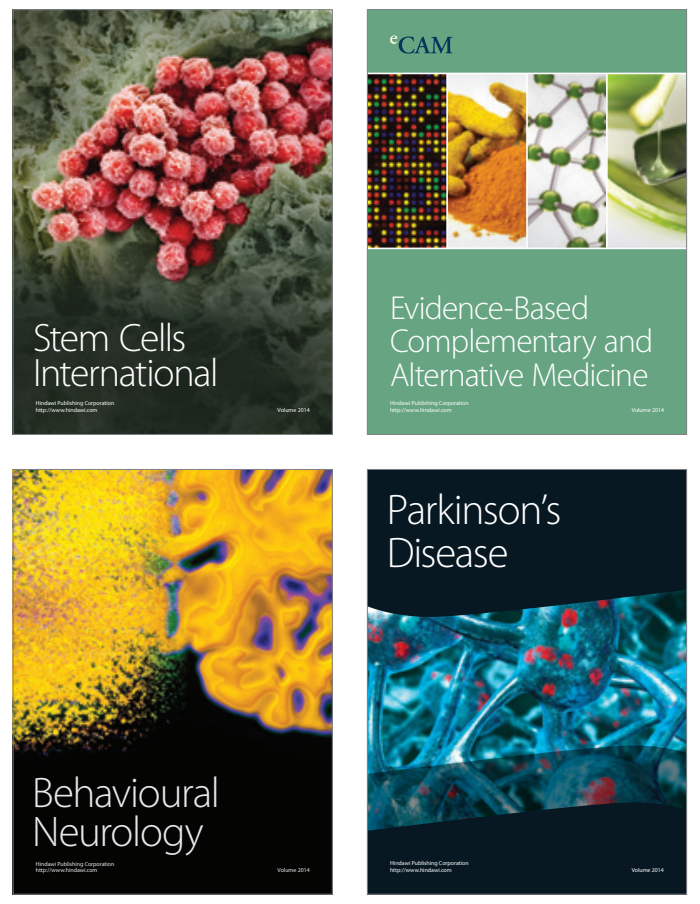
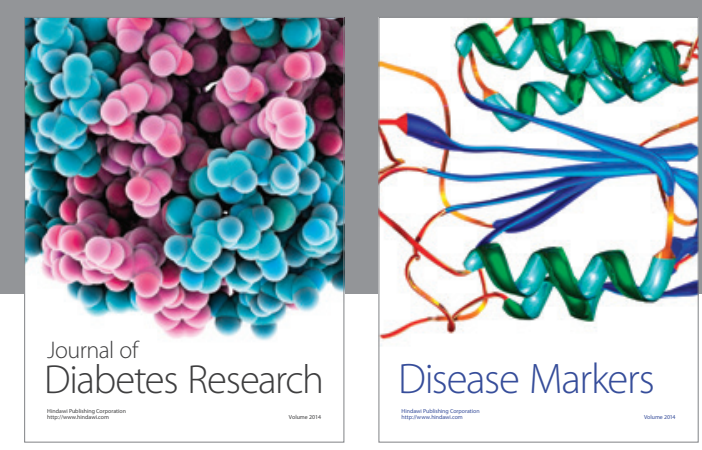

Disease Markers
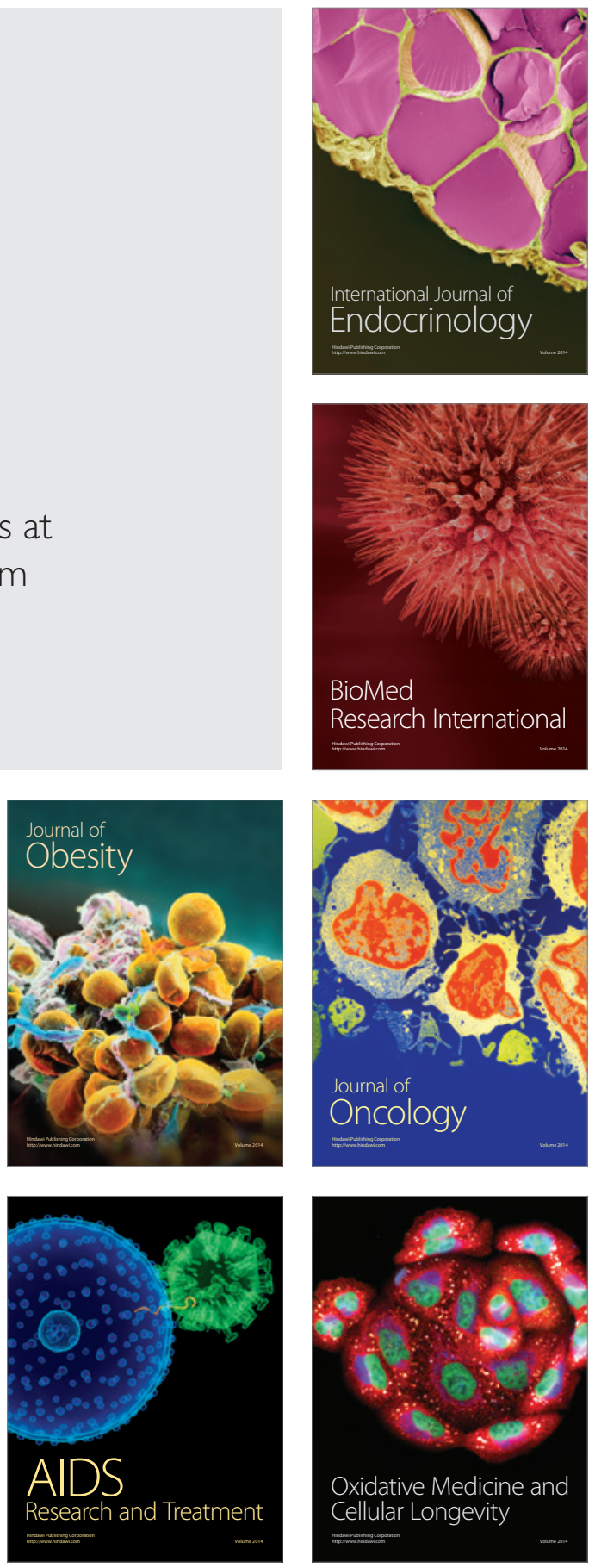